\section{LOCAN: A general FORTRAN program for latency-operating characteristic (LOC) analysis}

\section{GAROLD STASSER and DEREK W. SCHULTZ University of Illinois, Champaign, Illinois 61820}

A FORTRAN program to compute latency-operating characteristics (LOCs) is presented. Latency and accuracy scores from within-subject designs having equal cell frequencies and containing no missing data are appropriate. Output includes both latency measures (mean and median reaction times) and accuracy measures $\left(d^{\prime}, d^{\prime 2}, \log\right.$ odds, and $\log$ eta) for each latency band. The user determines the number of latency levels into which the data are partitioned. Input, output, and program subroutines are discussed.

It is well known that speed and accuracy trade off in most information processing tasks (Fitts, 1966; Garrett, 1922; Ollman, 1966). Wood and Jennings (1976) noted that analyses of such speed-accuracy tradeoff functions (SATFs) can be classified as manipulated-criterion or free-criterion designs. In the former, the experimenter either instructs the subject to respond within a predetermined time window or deadline period, which varies over experimental blocks or sessions, or varies a payoff schedule to manipulate the speed and accuracy of the subject's responses. Speed and accuracy of responding are then computed for each deadline or payoff condition. On the other hand, free-criterion examinations of SATFs have taken two forms. The first is the conditionalized accuracy function (CAF), as discussed by Wood and Jennings (1976) and Lappin (Note 1). CAFs are computed by partitioning a latency distribution into equal-latency bands (not necessarily composed of equal numbers of trials) and computing accuracy within each time band. The second form of free-criterion analyses is the latency-operating characteristic (LOC), as employed in the work of Lappin and Disch (1972a, 1973) and Lappin and Harm (1973; Harm \& Lappin, 1973), ${ }^{1}$ LOCs are computed by partitioning a latency distribution into bands of equal numbers of trials and computing accuracy and latency measures for each band.

Though various researchers have examined these SATF analyses, no single procedure has been widely adopted. Among micro-tradeoff functions, use of LOC analysis is most widespread. Moreover, current trends in

This work was supported in part by University of Illinois Research Board Grant 40-32-66-390 to Dr. Christopher Wickens and Derek Schultz and by NIMH Traineeship in Measurement Psychology MH 14257-02 to Garold Stasser. The authors would like to thank Joseph Lappin and Christopher Wickens for their helpful comments and suggestions. information processing research suggest that SATF analyses will become as commonplace as use of signal detection methodology. Finally, since computation of LOCs is simple in principle but tedious in practice, computers are ideal for LOC analysis. It is for these reasons that LOCAN was developed.

Brief Description of Program. LOCAN calculates the accuracy and reaction time (RT) summary statistics typically used in LOC analysis for both the individual subject's data and the combined data for all subjects. The data for each subject are ranked by RTs within each condition. The ranked data are then partitioned into the number of blocks specified by the user, and the mean and median RT and the accuracy indices $\left(\mathrm{d}^{\prime}, \mathrm{d}^{\prime 2}\right.$, $\log$ odds, $\log$ eta) are calculated for each block. After analyzing the individual subject's data separately, the program aggregates the data across respective latency blocks by condition and recalculates the RT and accuracy measures for each latency band on all subjects' data combined.

Since choice of a specific accuracy measure is still a matter of personal discretion, this program computes all commonly used statistics. Similarly, choice of mean or median RTs largely depends on the degree of skewness within the distribution of latencies. Thus, this choice is also left to the user.

Specific definitions of the accuracy indices computed by LOCAN are as follows:

$$
\begin{gathered}
\mathrm{d}^{\prime}=\mathrm{Z}_{\mathrm{P}_{\mathrm{C}}}-\mathrm{Z}_{\mathrm{P}_{\mathrm{E}}} \\
\log \text { odds }=\log _{10}\left(\mathrm{P}_{\mathrm{C}} / \mathrm{P}_{\mathrm{E}}\right) \\
\log \text { eta }=-\frac{1}{2} \log _{10}\left[\left(\mathrm{P}_{\mathrm{C} 1} / \mathrm{P}_{\mathrm{E} 1}\right) *\left(\mathrm{P}_{\mathrm{C} 2} / \mathrm{P}_{\mathrm{E} 2}\right)\right] .
\end{gathered}
$$

$\mathrm{Z}_{\mathrm{P}_{\mathrm{C}}}$ and $\mathrm{Z}_{\mathrm{P}_{\mathrm{E}}}$ are the unit normal deviates associated with the probability of a correct response, $\mathbf{P}_{\mathbf{C}}$, and the probability of an error, $P_{E}$, respectively. For the $\log$ eta index, $\mathbf{P}_{\mathrm{C}_{1}}\left(\mathbf{P}_{\mathrm{E} 1}\right)$ and $\mathrm{P}_{\mathrm{C} 2}\left(\mathbf{P}_{\mathrm{E} 2}\right)$ are the probabilities of a correct response (error) for Stimulus Instance 1 and for Stimulus Instance 2, respectively. Note that the calculation of the log eta index assumes that only two stimuli are used. To estimate the unit normal deviate, $Z$, associated with a given probability, $P$, we employed the approximation reported by Gulliksen and Tucker (Note 2). This approximation yields estimates of $\mathrm{Z}$ with a maximum discrepancy less than $3 \times 10^{-4}$ when $.02<\mathrm{p}<.98$. Accuracy measures (viz., $\mathrm{d}^{\prime}$ and $\mathrm{d}^{\prime 2}$ ) calculated using extreme probabilities of errors (i.e., less than .01 or greater than .99 ) may be severely biased.

LOCAN is written in FORTRAN IV G and can be run on any IBM 360 or IBM 370 ; in fact, the program would be compatible with most FORTRAN compilers. In its present version, standard peripheral hardware (viz., card reader and printer) are required. However, 
only minor modifications would permit the use of options such as tape or disk input or punched output. Furthermore, although the arrays are dimensioned to handle most data sets, adjustment in array sizes requires changing only the dimension statements in the main program since the arrays are dimensioned at run time in the subroutines as a function of the input parameters described below.

Input to LOCAN. Necessary input includes a usersupplied format, a parameter card, and the data. The first record of the input is a FORTRAN format sufficient to read in one subject's data for one condition. Thus, a user has a considerable flexibility in the actual form of the input data. The second record of the input specifies a set of parameters describing the current analysis. These parameters include the number of trials per condition, the number of experimental conditions, the number of subjects, and the number of blocks into which the data are to be partitioned for the LOC analysis. The third and all subsequent records are the data. The present version of LOCAN places the following restrictions on the form of the input data. First, the RT for each trial must be followed immediately either by an error code (Option 1) or by the obtained response and by the correct response (Option 2). The option used is indicated on the parameter card, and Option 2 must be used if log eta is desired as an accuracy index. Second, all the data for a subject must be presented consecutively with each condition starting on a new record. Third, the order of the experimental conditions must be the same for all subjects. Finally, since any missing trials are treated by LOCAN as correct trials of zero latency, no missing trials can be tolerated for an accurate analysis. Minor deviations from these requirements can be accommodated without extensive revision.

Output from LOCAN. The statistics for each subject and each condition are printed in a table with a row for each block and a column for each statistic. Such a table is also printed for each condition of the aggregate data. Headings specify the subject number (or "All Subjects" for the aggregate data) and the condition number for each table. (An available version of the program also allows the user to choose one of the accuracy statistics to be plotted as a function of either the mean or median RT on the University of Illinois Model 763 Calcomp installation. Minor revisions should allow plotting on other comparable plotting installations.)

In addition to the above output, LOCAN prints out the user-supplied parameters and the necessary array dimensions for the current analysis. This output allows the user to double-check the input parameters and necessary storage requirements for any particular application. Furthermore, the program checks for conditions which prohibit the calculation of any of the statistics (e.g., no errors in a block), prints a warning if such conditions exist, and performs a fix-up to permit continuation of the analysis.

LOCAN computes no dispersion indices (e.g., standard deviations, standard errors, or confidence intervals). However, such statistics are easily computed by the user. Similarly, though LOCAN does not directly compute centroids for each experimental condition, the user can obtain centroid statistics by partitioning the latency distribution into a single latency band (i.e., by setting the number of blocks at one). Subsequent analyses using the tabulated output from LOCAN (such as discriminant analysis, multivariate analysis of variance, and regression analysis) can be conducted through standard statistical packages. Similarly, segmental regression analysis, such as that described by Bongartz (1968), is not provided within LOCAN.

Availability. A card listing and detailed program description is available at no charge from the second author at 514 Psychology Building, University of Illinois, Champaign, Illinois 61820.

\section{REFERENCE NOTES}

1. Lappin. J. S. One test of the adjustable timing model for choice reaction time. Unpublished manuscript, Vanderbilt University, 1975.

2. Gulliksen, H., \& Tucker, L. R. An IBM 650 program for paired comparisons from balanced incomplete blocks: $A$ 6-31 design (Parcobib 6-31). (ETS RM 59-5). Princeton, N.J: Educational Testing Service, 1959.

\section{REFERENCES}

BongarTz, R. S. A least-squares method for fitting intercepting line segments to a set of data points. Psychological Bulletin, 1968, 70, 749-755.

Frrrs, P. M. Cognitive aspects of information processing: III. Set for speed versus accuracy. Journal of Experimental Psychology, 1966, 71, 849-857.

GARRET, H. E. A study of the relation of accuracy to speed. Archives of Psychology, New York, 1922, No. 56.

HARM, O. J., \& LAPPIN, J. S. Probability, compatibility, speed, and accuracy. Journal of Experimental Psychology, 1973, 100, 416-418.

LAPPIN, J. S., \& Disch, K. The latency operating characteristic: I. Effects of stimulus probability on choice reaction time. Journal of Experimental Psychology, 1972, 92, 419-427. (a)

Lappin, J. S., \& Disch, K. The latency operating characteristic: II. Effects of visual stimulus intensity on choice reaction time. Journal of Experimental Psychology, 1972, 93, 367-372. (b)

LAPPIN, J. S., \& Disch, K. The latency operating characteristic: III. Temporal uncertainty effects. Journal of Experimental Psychology, 1973, 98, 279-285.

LAPPIN, J. S., \& HARM, O. J. On the rate of acquisition of visual information about space, time, and intensity. Perception \& Psychophysics, 1973, 13, 439-445.

Ollman, R. T. Fast guesses in choice reaction time. Psychonomic Science, 1966, 6, 155-156. 
Wood, C. C., \& Jennings, R. J. Speed-accuracy trade-off functions in choice reaction time: Experimental designs and computational

Psychophysics, 1976, 19, 92-101.

\section{NOTE}

1. Theoretically, LOCs are graphic representations of a perceptual process parameterized by speed and accuracy and free from effects of the subject's decision criterion. As surh, LOCs have been presumed to be a reliable estimation of the rate of information transmission through a perceptual channel. However, this presumption is valid only to the extent that errors are assumed to arise from malfunctions in perceptual processing rather than in later stages of processing (e.g., response selection or execution).

(Accepted for publication June 10, 1977.) 